\title{
Características en alumnos de bajo rendimiento en la asignatura de Cálculo Diferencial en el ITSLP
}

\section{Characteristics in low-performing students in the subject of Differential Calculus in the ITSLP}

\author{
DOI: $10.46932 / s f j d v 3 n 1-054$
}

Received in: Jan 30st, 2021

Accepted in: Feb 1th, 2022

\section{Ángela Rebeca Garcés Rodríguez}

Química

Subdirectora de Servicios Administrativos, Docente del Departamento de Ciencias Básicas, Tecnológico Nacional de México / Instituto Tecnológico de San Luis Potosí.

Av. Tecnológico S/N, Unidad Ponciano Arriaga, 78437, Soledad de Graciano Sánchez, S.L.P.

E-mail: México.angela.gr@slp.tecnm.mx

\section{Gustavo Vera Reveles}

Doctor en Ciencias Aplicadas

Docente del Departamento de Ciencias Básicas, Departamento de Eléctrica, Electrónica y Mecatrónica, Tecnológico Nacional de México / Instituto Tecnológico de San Luis Potosí.

Av. Tecnológico S/N, Unidad Ponciano Arriaga, 78437, Soledad de Graciano Sánchez, S.L.P. México.

E-mail: gustavo.vr@slp.tecnm.mx

\section{Rutilo Moreno Monsiváis}

Maestro en Ciencias Aplicadas

Jefe de Proyectos de Vinculación, Docente del Departamento de Ciencias Básicas, Tecnológico

Nacional de México / Instituto Tecnológico de San Luis Potosí.

Av. Tecnológico S/N, Unidad Ponciano Arriaga, 78437, Soledad de Graciano Sánchez, S.L.P. México.

E-mail: rutilo.mm@slp.tecnm.mx

\section{María Eugenia Navarrete Sánchez}

Doctora en Educación

Docente del Departamento de Ciencias Básicas, Tecnológico Nacional de México / Instituto

Tecnológico de San Luis Potosí.

Av. Tecnológico S/N, Unidad Ponciano Arriaga, 78437, Soledad de Graciano Sánchez, S.L.P. México.

E-mail: maria.ns@slp.tecnm.mx

\section{Sergio Alberto Rosalío Piña Granja \\ Doctor en Educación}

Jefe de proyectos de Docencia, Docente del Departamento de Ciencias Básicas, Tecnológico Nacional de México / Instituto Tecnológico de San Luis Potosí.

Av. Tecnológico S/N, Unidad Ponciano Arriaga, 78437, Soledad de Graciano Sánchez, S.L.P. México.

E-mail: sergio.pg@slp.tecnm.mx

\section{Octavio Villalobos Fernández}

Maestro en Ciencias Aplicadas

Subdirector de Planeación y Vinculación, Docente del Departamento de Ciencias Básicas, Tecnológico Nacional de México / Instituto Tecnológico de San Luis Potosí. 
Av. Tecnológico S/N, Unidad Ponciano Arriaga, 78437, Soledad de Graciano Sánchez, S.L.P. México.

E-mail: octavio.vf@slp.tecnm.mx

\title{
María Laura Granja García
}

Maestría en Educación con especialidad en Docencia.

Jefa de Proyectos de Investigación, Docente del Departamento de Ciencias Básicas, Tecnológico

Nacional de México / Instituto Tecnológico de San Luis Potosí.

Av. Tecnológico S/N, Unidad Ponciano Arriaga, 78437, Soledad de Graciano Sánchez, S.L.P. México.

E-mail: maria.gg@slp.tecnm.mx

\section{Edmundo Cerda Rodríguez}

Doctor en Gestión Educativa

Docente del Departamento de Ciencias Básicas, Tecnológico Nacional de México / Instituto

Tecnológico de San Luis Potosí.

Av. Tecnológico S/N, Unidad Ponciano Arriaga, 78437, Soledad de Graciano Sánchez, S.L.P. México.

E-mail: edmundo.cr@slp.tecnm.mx

\section{RESUMEN}

La presente investigación muestra un análisis de las variables relacionadas respecto al bajo desempeño en estudiantes activos, los resultados obtenidos permiten identificar factores; académicos, de desempeño profesional y socio-cultural que pudiesen tener un impacto significativo, en que el estudiante con bajo desempeño académico, pudiese tomar la decisión de abandonar sus estudios profesionales, nuestro principal interés está relacionado en aquellos estudiantes que se encuentran cursando la materia de Calculo Diferencial por tercera ocasión, contrastando con los que la cursan por segunda y primera ocasión, en el Instituto Tecnológico de San Luis Potosí (ITSLP). Complementando el análisis con el promedio obtenido en la preparatoria y en el Examen Ceneval, para el ingreso a la educación superior.

Palabras clave: Deserción, bajo desempeño, educación superior, Cálculo Diferencial.

\begin{abstract}
The present research shows an analysis of the variables related to low performance in active students, the results obtained allow us to identify factors; academic, professional performance and socio-cultural that could have a significant impact, in which the student with low academic performance, could make the decision to abandon their professional studies, our main interest is related to those students who are taking Differential Calculus for the third time, contrasting with those who are taking it for the second and first time, at the Instituto Tecnológico de San Luis Potosí (ITSLP). Complementing the analysis with the average obtained in high school and in the Ceneval exam, for the entrance to higher education.
\end{abstract}

Keywords: Desertion, low performance, higher education, Differential Calculus.

\section{INTRODUCCIÓN}

Las cifras consultadas en el Programa Institucional de Innovación y Desarrollo (PIID) 20132018, indican que en el ciclo escolar 2012-2013 se registró un 54.33\% de eficiencia terminal en todas las instituciones que formaban el entonces Sistema Nacional de Institutos Tecnológicos, ahora Tecnológico Nacional de México (TecNM), dado lo anterior, es importante para el TecNM indagar las 
causas por las que cerca de la mitad de sus alumnos no logra concluir sus estudios a tiempo. El plan nacional de desarrollo 2013-2018 en el apartado México con Educación de Calidad menciona, que la falta de educación es una barrera para el desarrollo productivo del país, ya que limita la capacidad de la población para comunicarse de una manera eficiente, trabajar en equipo, resolver problemas, usar efectivamente las tecnologías de la información para adoptar procesos y tecnologías superiores, así como para comprender el entorno en el que vivimos y poder innovar. Se ha detectado que la deserción responde a una multiplicidad de factores que afectan a los estudiantes (De los Santos, 1993 \& Martínez Rizo, 1989), entre ellos, se encuentran las características académicas previas del estudiante, tales como los bajos promedios obtenidos en la educación media superior, la insuficiencia de los conocimientos y habilidades con que egresan los estudiantes de este nivel para atender las exigencias académicas del nivel superior, principalmente durante el primer año posterior a su ingreso a la licenciatura.

Es posible suponer, con cierto grado de certeza, que los estudiantes que ingresan a la educación superior no dominan las habilidades ni la información y conocimientos indispensables para utilizar, de la mejor manera posible, los recursos que la universidad pone a su disposición, muchos jóvenes no siguen los estudios porque prefieren encontrar un empleo a temprana edad para ayudar a sus familias, y las becas para este objetivo aún son escasas, señala el "Informe sobre la educación superior en América Latina y el Caribe" de la Unesco. (2005).

Cada minuto renunciaron a la escuela 2.2 niños y jóvenes mexicanos, según los datos del documento (principales cifras 2015-2016, de estadística educativa de la SEP), para David Calderón, presidente de Mexicanos Primero, el problema empieza desde la educación básica, puesto que en la secundaria los jóvenes no reciben la orientación necesaria para tomar una buena decisión con respecto a su bachillerato, que les ofrezca calidad (Moreno, T (2017, 21 Agosto) El Universal). En España, Jano y Ortiz (2005), en el trabajo titulado "Determinación de los factores que afectan al rendimiento académico en la educación superior", han investigado los factores, llegando a la conclusión de que uno de ellos es el esfuerzo efectivo realizado por el estudiante, así como sus habilidades y conocimientos previos a su ingreso a la universidad. Se han encontrado algunas variables que se asocian al rendimiento académico de los estudiantes. La primera de ellas ha sido la del deseo de estudiar un posgrado, mostrando que aquellos alumnos que han tenido mayor puntuación en dicha variable, logran mejores calificaciones en su carrera, lo cual parece concordar con lo que afirman Jano y Ortiz (2005) y Moreno, en el sentido de que los que obtienen mayores calificaciones son aquellos que dedican un mayor esfuerzo a las actividades de estudio. Asimismo, los estudiantes que han logrado mejores promedios al cursar el nivel del bachillerato, obtienen también mejores promedios en su licenciatura, lo cual concuerda con el caso de la Universidad 
de Costa Rica (2007), Torres Torres N. (2022) en su estudio de tesis llega a la conclusión que las estrategias didácticas se relacionan con la comprensión lectora de los estudiantes.

Arias y Flores (2005) en la Universidad Veracruzana, Pascarella y Terenzini (1983) en Estados Unidos, mencionan que las estudiantes del género femenino obtienen mejores calificaciones que sus colegas del género masculino.

Según datos de Cifras significativas 2016-2017, de los 3762679 estudiantes que cursan nivel superior, la SEP reporta un 6.8\% de abandono escolar, lo cual corresponde a 255862 estudiantes desertores.

En el estado de San Luis Potosí, la estadística educativa reporta 76,081 alumnos que cursan el nivel superior en el mismo ciclo ya mencionado, con una tasa de deserción del $10 \%$, correspondiente a 7608 estudiantes desertores.

Particularmente nuestro objeto de estudio, según datos del SII (Sistema Integral de Información) del Instituto Tecnológico de San Luis Potosí, reporta de un total de 1135 estudiantes que ingresaron en los periodos enero-junio 2012 y agosto diciembre 2012, con terminación de la carrera a 10 semestres, correspondientes a los periodos enero-junio 2016 y agosto-diciembre 2016, indica un porcentaje de deserción escolar del $39.11 \%$. Derivado de los datos obtenidos en porcentaje de deserción, dentro del Instituto Tecnológico de San Luis Potosí queda la siguiente interrogante: ¿Cuáles son los principales factores que determinan la falta de motivación en particular dentro del ITSLP, que puede conllevar a abandonar los estudios de nivel superior?

\section{DESCRIPCIÓN DEL MÉTODO}

La metodología utilizada en esta investigación es de dos tipos: la primera es de tipo descriptiva, se aplicó una investigación de campo, con una encuesta aplicada a una muestra de 50 alumnos de formación básica en el período escolar de Agosto-Diciembre del 2017, que cursaron la materia de cálculo diferencial por tercera ocasión a los cuales les denominamos alumnos con bajo rendimiento académico, la segunda es de tipo cuasi-experimental, se aplicó la misma encuesta a una población mayor de 50 alumnos que cursan la misma materia pero por $2^{\mathrm{a}}$ ocasión, el universo de estudio fueron los alumnos de 1er a 3er semestre de las distintas carreras ofrecidas por la Institución, la información se recabó mediante encuestas a los estudiantes que tuvieron disposición para responder. Las variables tomadas para la encuesta tienen su sustento en la bibliografía de: DIPLADI-UAM, (abril de 1987), De Vries, Wietse (2011).

Además, la investigación es tanto cualitativa, por la interpretación de los distintos factores en el bajo desempeño escolar, como cuantitativa, ya que se analizaron los datos obtenidos de las encuestas, de los promedios en la educación media superior, del examen de ingreso a la educación 
superior y del Sistema Integral de Información Institucional.

\subsection{POBLACIÓN Y MUESTRA}

Para la determinación del tamaño de muestra, se tomó como grupo de estudio a 50 estudiantes de nuevo ingreso que llevan la asignatura de Cálculo Diferencial del periodo Agosto-Diciembre 2017, otros grupos de 130 y 82 estudiantes, que llevan la asignatura por segunda y tercera vez respectivamente, se hizo uso de la fórmula clásica de Aguilar Barojas, S.(2005) con un intervalo de confianza del 95\%, un porcentaje de error del $10 \%$, siendo justa en el segundo caso y estando sobrada para el tercer caso. Los resultados de las encuestas fueron analizados con el programa estadístico SPSS IBM, la encuesta aplicada contempla un total de 34 preguntas, de ellas, 20 tienen formato de escala Likert y se refieren a factores personales como institucionales. En este trabajo, se presentan en gráficos y tablas específicas, factores: académicos, de desempeño profesional y socio-cultural más representativos de la encuesta aplicada, que nos ayudan a visualizar tendencias e inclinaciones según la oportunidad que cursan los estudiantes.

\section{RESULTADOS Y DISCUSIÓN}

La encuesta fue contestada por tres grupos diferentes, cada uno de 50 estudiantes, que llevaron la materia de Cálculo Diferencial, los cuales se indican como: primera, segunda y tercera oportunidad, derivado de que se están comparando 3 grupos y cada reactivo puede llegar a tener más de 4 variables, se representarán en la mayoría de los gráficos las dos frecuencias porcentuales más altas de cada grupo de estudiantes, en algunos casos de forma cuantitativa, en otros casos de forma cualitativa, las demás variables también fueron obtenidas, pero no se presentan en este trabajo, derivado de que no se observa información estadísticamente significativa que pudieran aportar una tendencia o conclusión en su análisis cualitativo o cuantitativo, según sea el caso.

La figura 1, muestra el promedio que el estudiante obtuvo en el nivel medio superior o equivalente, se puede observar una tendencia lineal a la baja, en función de si el estudiante lleva la asignatura en primera, segunda o tercera oportunidad. Ahondando sobre el tema y analizándolo se puede observar que, en cada una de las oportunidades, el género femenino obtiene mejor calificación que el masculino. 
Figura 1. Promedio en educación media superior o equivalente por número de oportunidad y por género.

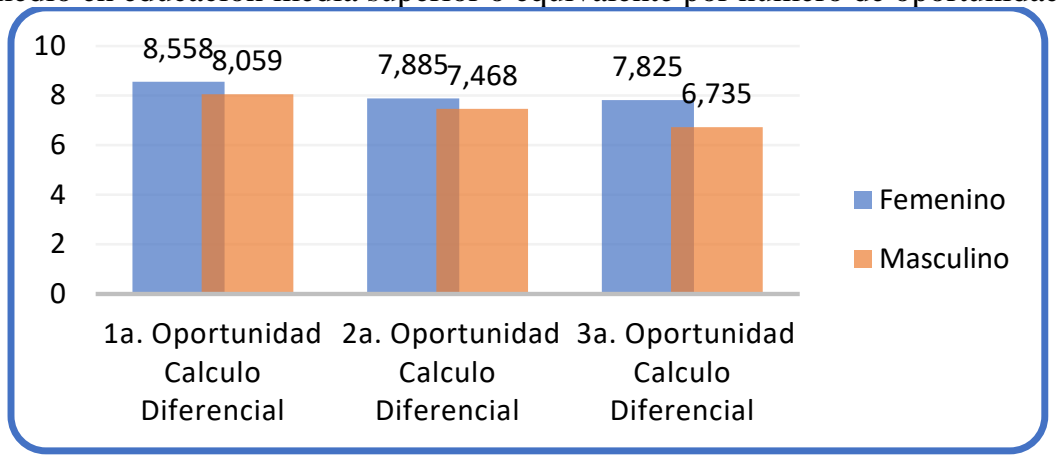

La puntuación en el examen Ceneval Exani II, es 700 puntos la más baja, y 1300 puntos la más alta, se puede observar que los alumnos de primera oportunidad tienen alrededor de 100 puntos de diferencia respecto a sus compañeros de segunda y tercera oportunidad, aunque tal vez se pudiera esperar un efecto de tendencia a la baja como la gráfica de promedio en el nivel medio superior, se puede observar que el grafico baja y luego sube, lo cual lo relacionamos directamente a que 8 alumnos que llevaron la materia por tercera oportunidad fueron alumnos que ya habían cursado la carrera en la misma Institución, y optaron por cursar otra diferente, posiblemente ya contaban con conocimientos necesarios para tener un mejor desempeño en su examen Exani II.

Figura 2. Promedio en examen Ceneval Exani II, por número de oportunidad y por género.

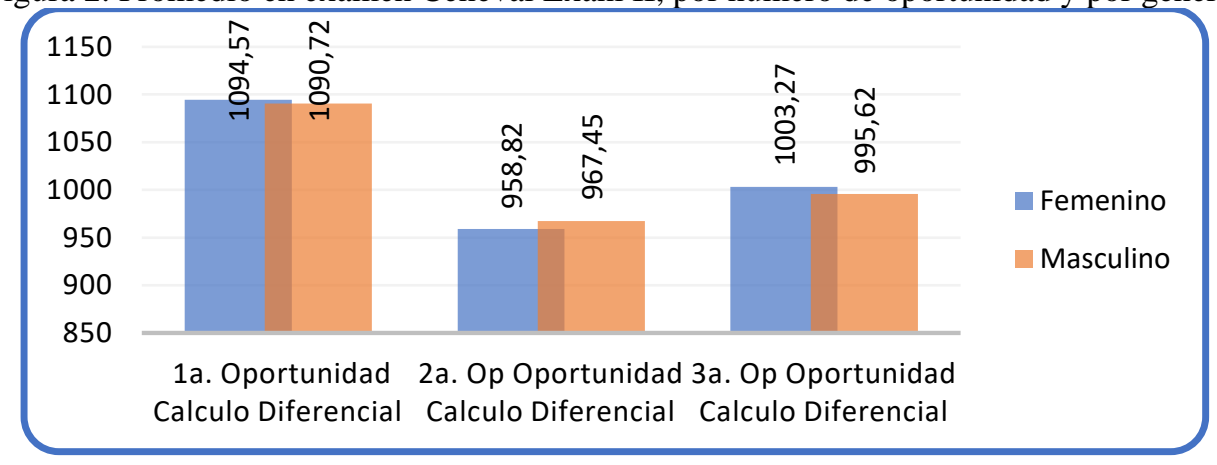

La máxima escolaridad de los padres, de los alumnos encuestados indica que, en los tres grupos de estudio, e invariantemente del género, en mayor porcentaje cuentan con estudios de preparatoria o equivalente. 
Figura 3. Máxima escolaridad de los padres.

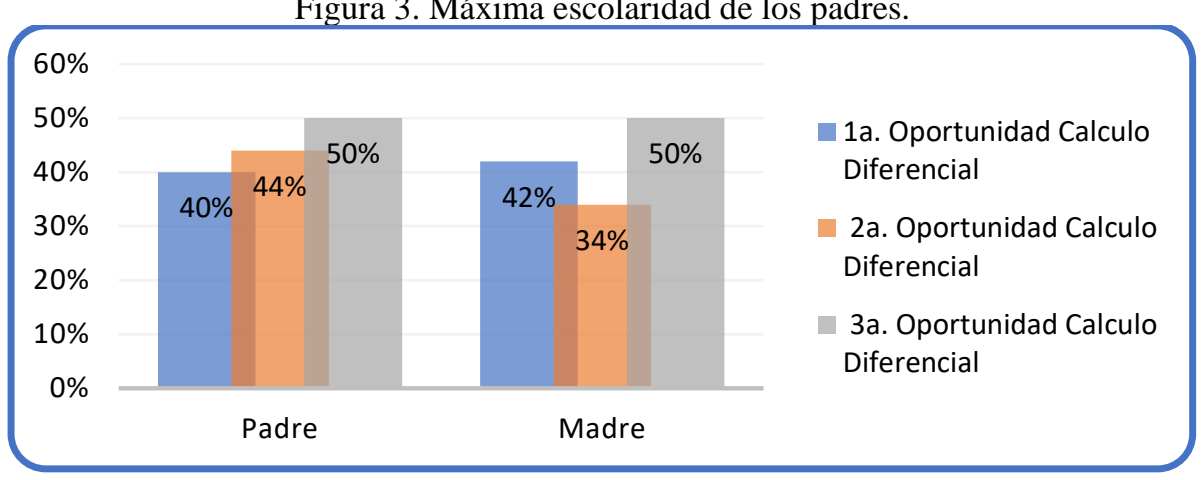

En lo referente a la pregunta acerca de cómo sienten los estudiantes que fue su desempeño en el nivel medio superior, los resultados son como muestra la figura 4, observando que, el promedio de los estudiantes de todas las oportunidades, arriba del $50 \%$ cuentan con la certeza que obtuvieron un buen desempeño en su educación media superior (este es un problema común relacionado a la falsa ilusión de competencia), seguido por un desempeño regular.

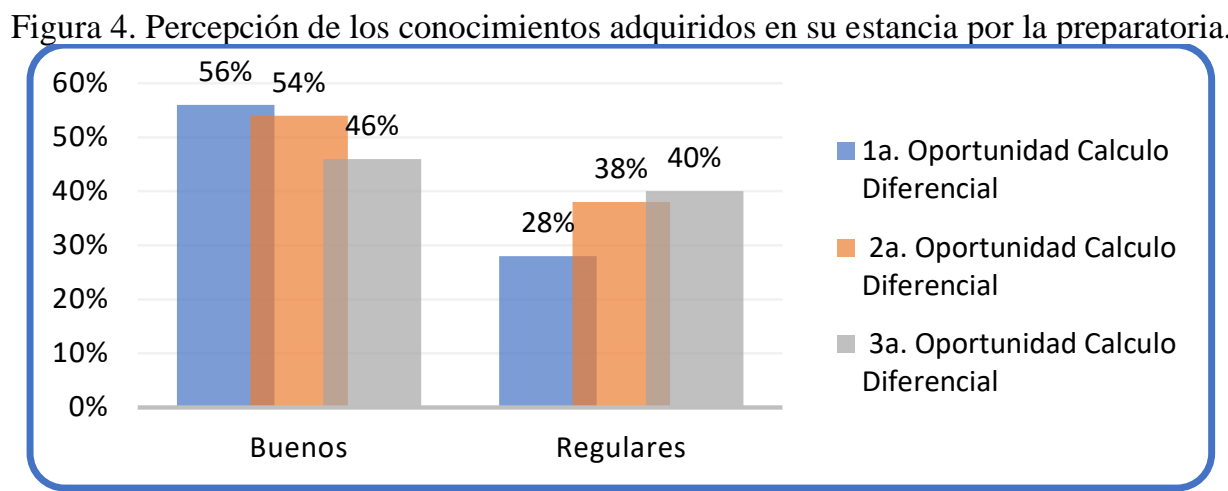

Sobre la disponibilidad de horarios según la figura 5, los que llegan a tener más problema con la elección de horario, son aquellos estudiantes que llevan la materia por tercera ocasión.

Figura 5. Disponibilidad de cursos según horarios.

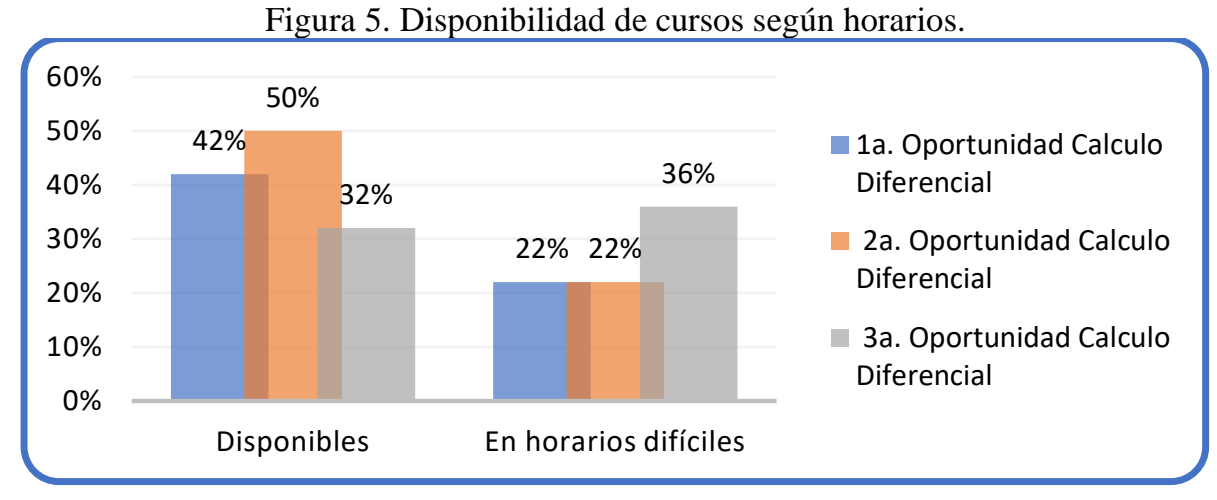

La figura 6 muestra que cerca del $60 \%$ del total de los estudiantes encuestados, mencionan tener hábitos de estudio. 
En la figura 7, cerca de la cuarta parte de los alumnos encuestados mencionan tener un disgusto por estudiar, según los gráficos mostrados con anterioridad, se podría esperar que los alumnos que les disgusta estudiar, fueran los que llevan la materia por tercera ocasión, sin embargo, los que mencionan tener un disgusto más alto por estudiar son los que llevan la materia por primera vez.
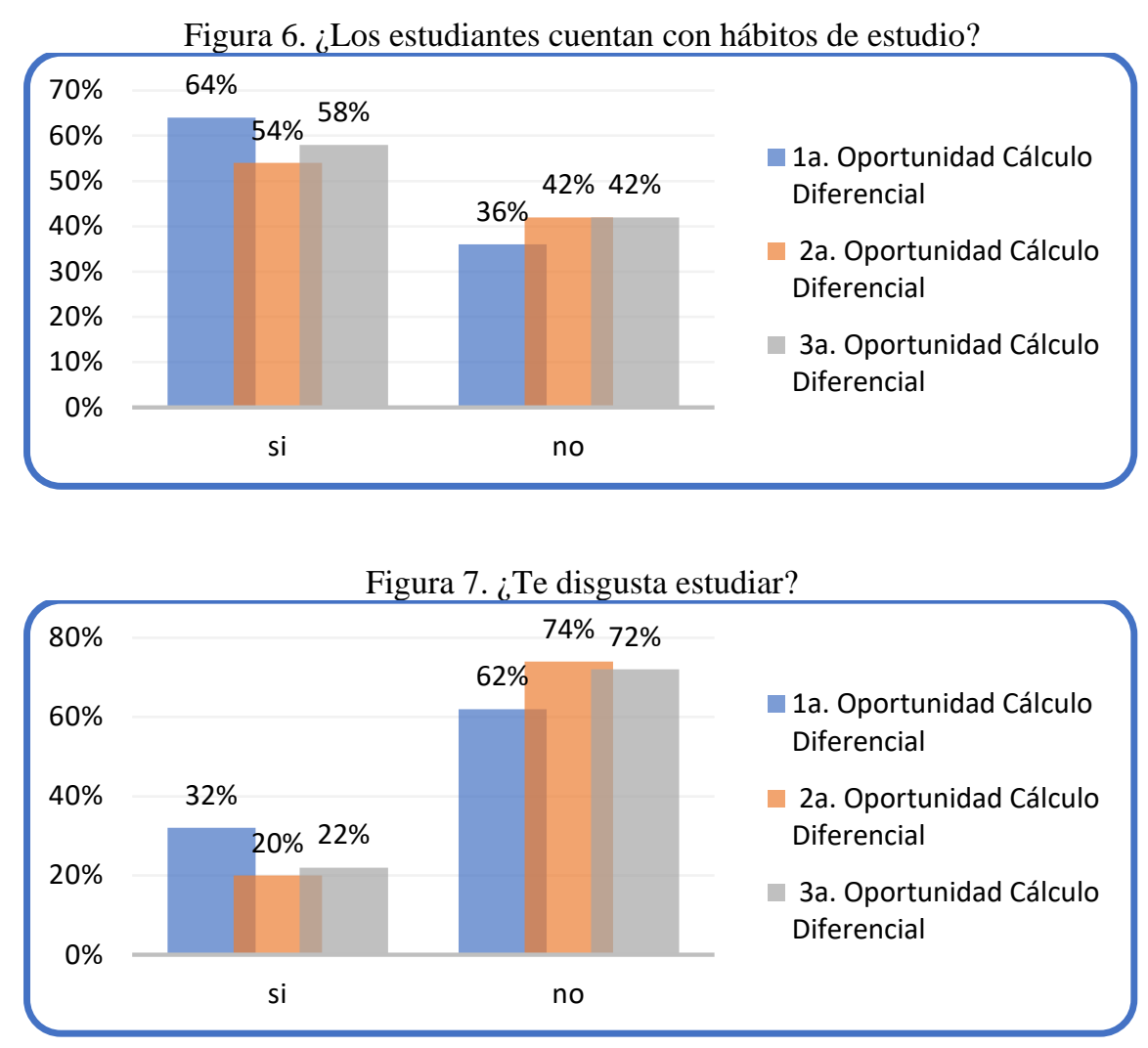

La tabla 1, menciona las horas que trabajan los estudiantes encuestados según la oportunidad que cursan la materia, se puede observar que los estudiantes de primera oportunidad trabajan el 30\%, de segunda oportunidad el $32 \%$, y los de tercera oportunidad lo hace el $50 \%$.

Tabla 1. Horas de trabajo, es visible que los estudiantes que llevan la materia por tercera ocasión, doblan en porcentaje los que lo hacen en el rango de 21 a 30 horas, con respecto a los que cursan la materia por primera y segunda oportunidad.

\begin{tabular}{|c|c|c|c|}
\hline Horas a la semana & \% Primera Oportunidad & \% Segunda Oportunidad & \% Tercera Oportunidad \\
\hline $1-10$ & 8 & 8 & 0 \\
\hline $11-20$ & 4 & 4 & 10 \\
\hline $21-30$ & 14 & 16 & 32 \\
\hline $31-40$ & 4 & 4 & 8 \\
\hline
\end{tabular}

El promedio que labora un estudiante que lleva la asignatura por primera vez es de 20.16 horas, en el segundo grupo, este promedio es de 20.5 horas, mientras que en el tercer grupo es de 25.1 horas, otro aspecto importante de analizar (de acuerdo al porcentaje de estudiantes que trabajan) es el motivo por el que lo hacen, lo cual podemos ver ilustrado en la Tabla 2. 
Tabla 2. Motivos por los que los estudiantes se ven en la necesidad de trabajar, es visible que los estudiantes que llevan la materia por tercera ocasión, por poco, doblan en porcentaje en el rubro de que los estudiantes trabajan para solventar gastos propios, con respecto a los que cursan la materia por primera y segunda oportunidad.

\begin{tabular}{|l|c|c|c|}
\hline Motivo de trabajo & \% Primera Oportunidad & \% Segunda Oportunidad & \% Tercera Oportunidad \\
\hline Situación Complicada en el hogar & 4 & 6 & 6 \\
\hline Obtención de Ingresos extra & 2 & 4 & 0 \\
\hline Solventar gastos propios & 20 & 22 & 40 \\
\hline Trabajo interno familiar & 4 & 0 & 4 \\
\hline
\end{tabular}

El promedio en porcentaje de estudiantes que reportan vivir con sus Padres/Familia de los países reportados en la tabla 3 , con respecto a alumnos de bajo desempeño académico en el ITSLP es inferior un $30.5 \%$, superior un $22.5 \%$ de los que reportan vivir independientemente, y $9 \%$ superior respecto a los que reportan que trabajan, cabe señalar que trabajar es la característica más común entre los países citados. Tabla según De Vries, Wietse (2011)

Tabla 3. Características que tienen en común estudiantes de otros países con nuestros estudiantes en $\%$.

\begin{tabular}{|l|c|c|c|c|c|}
\hline \multicolumn{7}{|c|}{ Características en común con otros Países } \\
\hline Características & Alemania & Francia & Países Bajos & Canadá & Bajo desempeño ITSLP \\
\hline Vive con Padres/Familiares & 24 & 46 & 45 & 51 & 72 \\
\hline Vive independientemente & 61 & 39 & 21 & 49 & 12 \\
\hline Trabaja & 66 & 48 & 77 & 45 & 50 \\
\hline
\end{tabular}

\section{CONCLUSIONES}

Como resultado encontramos que dentro de los factores que afectan el rendimiento escolar son: En variables académicas; el esfuerzo efectivo realizado por el estudiante, sus habilidades y conocimientos previos a su ingreso a la universidad, al menos los estudiantes relacionados a esta investigación que llevaron la materia de Calculo Diferencial por $2^{\mathrm{a}}$ y $3^{\mathrm{a}}$ ocasión, llegan a la Institución con deficiencias educativas, esto lo vemos directamente relacionado con el promedio obtenido en la educación media superior y en el examen de ingreso a la educación superior, la máxima escolaridad de los padres en todas las oportunidades fue Preparatoria, no observamos un cambio significativo en los promedios obtenidos derivado de dicha escolaridad. Las mayores frecuencias porcentuales de alumnos en todas las oportunidades indican haber obtenido un buen desempeño en su educación media superior, contar con hábitos de estudio así como el gusto por estudiar; en los estudiantes de segunda y tercera oportunidad pudiera tratarse de una falsa ilusión de competencia, ya que el estudiante tiene la creencia que su esfuerzo y promedio son buenos comparados con el resto de sus compañeros, también se corrobora la teoría en este estudio a que las estudiantes del género femenino obtienen mejores calificaciones que sus colegas del género masculino, lo cual vemos reflejado en este estudio, el $91 \%$ del total de alumnos encuestados en todas las oportunidades indican que la carrera que cursan es la que deseaban. En variables de desempeño profesional indican en relación a las horas de trabajo, los alumnos que llevan la materia por tercera ocasión el $50 \%$ trabajan medio tiempo, indicando que el motivo principal es el de poder solventar sus gastos 
propios, disminuyendo así el tiempo que le podrían dedicar a reforzar sus conocimientos atrasados y los actuales. Por ultimo respecto a variables de desarrollo personal y socio cultural el $80 \%$ de los alumnos encuestados de todas las oportunidades indican que el medio ambiente dentro del Instituto es muy agradable, así como que sienten lograr identificarse con la gente que estudia y labora en la Institución. 


\section{REFERENCIAS}

Aguilar Barojas, S. Fórmulas para el cálculo de la muestra en investigaciones de salud. Salud en Tabasco [en linea] 2005, 11 (enero-agosto):

Disponible en:<http://www.redalyc.org/articulo.oa?id=48711206> ISSN 1405-2091.

Arias, F. \& Flores, M. A. (2005). La satisfacción de los estudiantes con su carrera y su relación con el promedio y el sexo. El caso de la carrera de Contaduría de la Universidad Veracruzana en Nogales, Veracruz. Hitos de Ciencias Económico Administrativas, 29(1), 9-14.

De los Santos V., J. Eliézer. (2003) "Los procesos de permanencia y abandono escolar en educación superior”. En Revista Iberoamericana de Educación No. 33. España, OEI. www.oei.es

De Vries, Wietse. León Arenas, P. Romero Muñoz, J. \& Hernández Saldaña, I. (2011). ¿Desertores o decepcionados? Distintas causas para abandonar los estudios universitarios. Revista de educación superior issn 0185-2760, vol xl(4), no. 160 pp.29-50.

González, L. (2006). "Repitencia y deserción universitaria en América Latina”, en: UNESCO-IESALC (2006). Informe sobre la educación superior en América Latina y el Caribe. 2000-2005. La metamorfosis de la educación superior, Caracas UNESCO-IESALC, pp. 156-168.

Izar, L. Cortés, Y. \& López G. (2011). Factores que afectan el desempeño académico de los estudiantes de nivel superior en Río Verde Revista de Investigación Educativa [en línea]. San Luis Potosí, México, 12, 35-56. Extraído el 8 mayo, 2012 de http://www.uv.mx/cpue/num12/opinion/completos/Izardesempeno\%20academico.pdf

Jano, D. \& Ortiz, S. (2005). Determinación de los factores que afectan al rendimiento académico en la educación superior. XII Jornadas de la Asociación de Economía de la Educación, Oviedo.

Martínez-Rizo, F. (1988) "Estudio de la eficiencia en cohortes aparentes”. Deserción, Rezago y Eficiencia Terminal en las IES. Propuesta metodológica para su estudio.

Montero, E. Villalobos, J. \& Valverde, A. (2007). Factores Institucionales, Pedagógicos, Psicosociales y Sociodemográficos asociados al Rendimiento Académico en la Universidad de Costa Rica: Un Estudio Multinivel. RELIEVE, 13(2), 215-234.

Moreno, T. (2017, 21 Agosto) El Universal: http://www.eluniversal.com.mx/nacion/sociedad/desercionescolar-infrenable-sep

Pascarella, E. \& Terenzini P. (1983). Predicting voluntary freshman year pesistence/withdrawal behavior in a residential university: a path analytic validation of Tinto's model. Journal of Educational Psychology, 75(2), 215-226.

Principales Cifras del Sistema Educativo Nacional 2016-2017 (2017).

http://www.planeacion.sep.gob.mx/Doc/estadistica_e_indicadores/principales_cifras/principales_cifras_ 2016_2017_bolsillo.pdf

TecNM, (2014). Programa Institucional de Innovación y Desarrollo 2013-2018. Tecnológico Nacional de México. Disponible en: http://www.tecnm.mx/images/areas/planeacion/2014/PIID_20132018_TECNM_Final.pdf. 
Torres Torres, N. Balladares Atoche C. Jácome Vera A. \& Salavarria Barco B. Estrategias didácticas y comprensión lectora en educación básica, Vol. 3 No. 1 (2022): South Florida Journal of Development, Miami, p.75-89 v. 3, n. 1, jan./feb. 2022.

Universidad Autónoma Metropolitana. “Análisis cuantitativo de la deserción escolar”, DIPLADI-UAM, México, octubre de 1988, 250 p. 